\title{
Allograft reconstruction for large parosteal osteoma of the clavicle: a case report
}

\author{
Donghyup Shin, Wonseok Kim, Jungho Park \\ Department of Orthopedic Surgery, Korea University Ansan Hospital, Ansan, Korea
}

\begin{abstract}
A large parosteal osteoma arising on the surface of the right clavicle of a 39-year-old male patient was suspected preoperatively as a parosteal osteosarcoma. The lesion was treated with wide resection and allograft reconstruction. In this case report, we discuss the accurate diagnosis and appropriate surgical treatment for unusual clavicular tumors.
\end{abstract}

Keywords: Clavicle; Osteoma; Allograft; Case report

Primary clavicle tumors are rare. Due to the rarity of clavicle tumors, accurate clinical diagnoses are difficult. Tumors arising from the surface of the clavicle such as parosteal osteomas or osteosarcomas are very rare. In a review of 206 cases published from 1980 to 2011 in East Asia, osteomas and osteosarcomas comprised $3.88 \%$ (8 cases) and $8.74 \%$ (18 cases) of cases, respectively, which was more than twice the prevalence of parosteal osteoma [1]. The authors report a case of a large parosteal osteoma of the clavicle treated with wide excision and allograft reconstruction due to the possibility of parosteal osteosarcoma based on the preoperative evaluation.

\section{CASE REPORT}

A 39-year-old male patient visited our hospital due to the presence of a right medial clavicular mass. A $3 \times 2-\mathrm{cm}$-sized, fixed, non-tender, hard, bony mass in the medial clavicle area was observed. Upon examination, Tinel's sign was negative with full range of motion in the right shoulder. This lesion had been identified 10 years prior and gradually had grown in size, but the patient had not undergone any treatment. A thorough evaluation was recommended after $\mathrm{x}$-ray examination, but the patient refused and did not return to the hospital. After 11 months, the patient revisited our hospital requesting removal of the mass. The lesion was larger than when it was initially found. His right elbow had a history of injury and exhibited a cubitus varus deformity with limitation of motion $\left(40^{\circ}-90^{\circ}\right)$ and ulnar nerve symptoms in the right hand. Deterioration in general conditions such as weight loss or lethargy was not observed. On X-ray, computed tomography (CT) scan, and contrast-enhanced magnetic resonance imaging (MRI), the mass measured $6.0 \mathrm{~cm}$ mediolaterally and $4.8 \mathrm{~cm}$ anteroposteriorly in the medial clavicle area (Fig. 1). The MRI revealed a lesion with low signal intensity on both T1and T2-weighted images. The lesion was composed of a dense osseous portion, and signal change was observed with suspected involvement of the adjacent subclavius muscle not involving the

Received: August 31, $2021 \quad$ Revised: October 8, $2021 \quad$ Accepted: October 8, 2021

Correspondence to: Jungho Park

Department of Orthopedic Surgery, Korea University Ansan Hospital, 123 Jeokgeum-ro, Danwon-gu, Ansan 15355, Korea

Tel: +82-31-412-4941, Fax: +82-31-487-9502, E-mail: canall@korea.ac.kr, ORCID: https://orcid.org/0000-0002-0641-8307

Financial support: None.

Conflict of interest: None.

Copyright@ 2021 Korean Shoulder and Elbow Society.

This is an Open Access article distributed under the terms of the Creative Commons Attribution Non-Commercial License (http://creativecommons.org/licenses/by-nc/4.0/) which permits unrestricted non-commercial use, distribution, and reproduction in any medium, provided the original work is properly cited. 


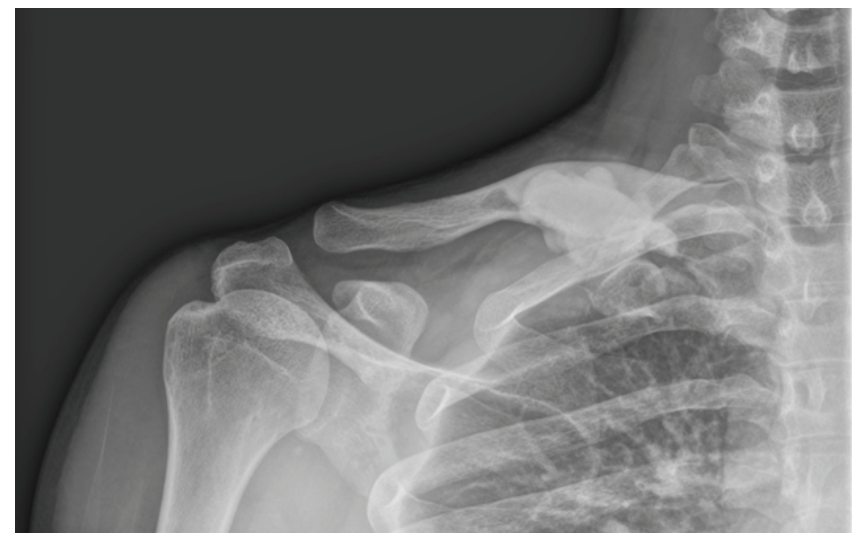

Fig. 1. On plain X-ray, a large dense sclerotic mass was noted in the area of the right medial clavicle.

first rib (Fig. 2). Through a multi-disciplinary approach with our radiology and hemato-oncology departments, the most likely diagnosis was parosteal osteosarcoma, followed by parosteal osteoma. The main reason for suspecting malignancy was involvement of the subscapularis enhancing soft tissue portion and deviating subclavian vein. To rule out malignancy and to determine the stage of tumor, chest CT, bone scan, and positron emission tomography (PET)-CT were performed. On the bone scan, an active lesion was identified on the right medial clavicle. No metastatic lesions were observed on PET-CT. After radiologic interpretation, the patient requested complete removal of the mass. Wide resection with allograft reconstruction was decided upon after extensive communication with the patient due to the large size of the mass, recent rapid growth, fear of recurrence with suspicious malignancy, and possibility of inappropriate diagnosis after inadequate biopsy. Medial resection was performed through the sternoclavicular joint, and lateral resection was created with a margin approximately $3 \mathrm{~cm}$ from the most lateral aspect of the clavicular mass (Fig. 3). During resection, soft tissue adhesion was identified between the posterior clavicle and the first rib. Atypical cells were not observed on frozen biopsy that included medial and lateral portions of the fist rib and subclavius muscle. Fibular allograft bone was inserted through the defect site after measuring the exact size. Fixation was performed between the sternum and fibular allograft and between the fibular allograft and remnant lateral clavicle using two plates and screws (Fig. 4). An additional plate was inserted between the two previously inserted plates to prevent stress fracture. Demineralized bone matrix was used to promote bone healing. After the operation, daily teriparatide (Forsteo; Lilly, Seoul, Korea) was injected subcutaneously for eight weeks. Five months after the operation, bony union was observed between the lateral strut and remnant clavicle on the follow-up X-ray. After 11 months, the patient visited
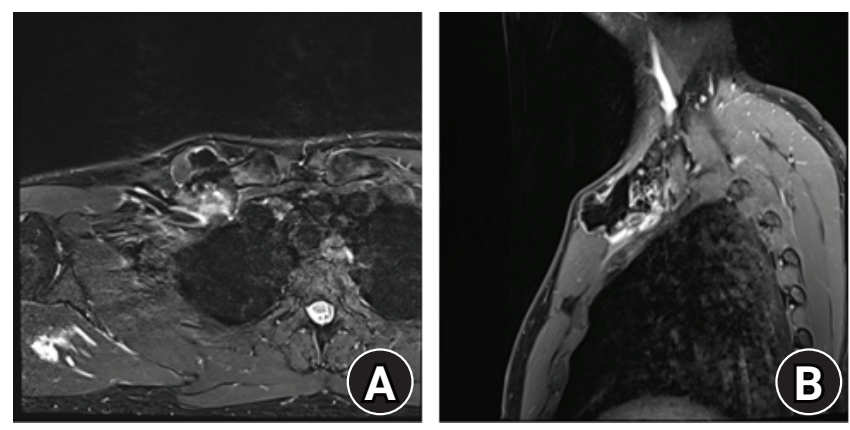

Fig. 2. Magnetic resonance imaging showing a lesion with low signal intensity on both T1- and T2-weighted images with suspected involvement of the adjacent subclavius muscle with enhancing soft tissue portion and deviating subclavian vein. (A) Axial image. (B) Sagittal image.

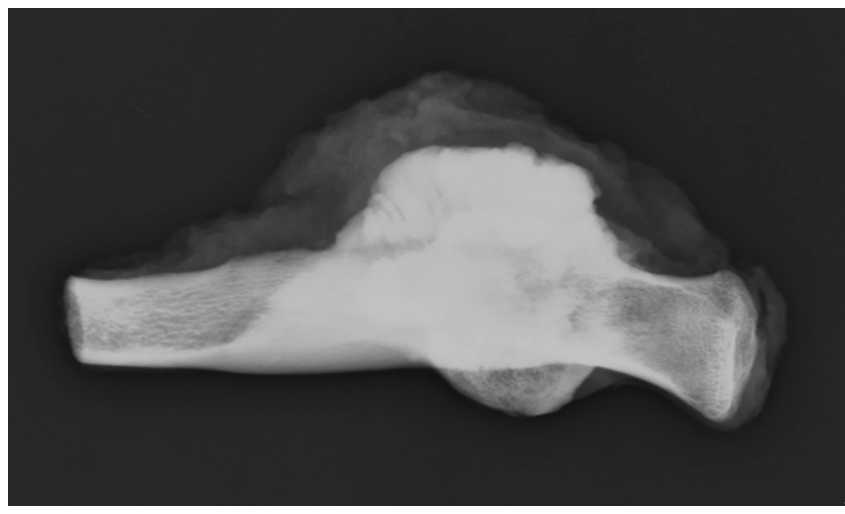

Fig. 3. A photograph of the resected clavicle including the entire mass.

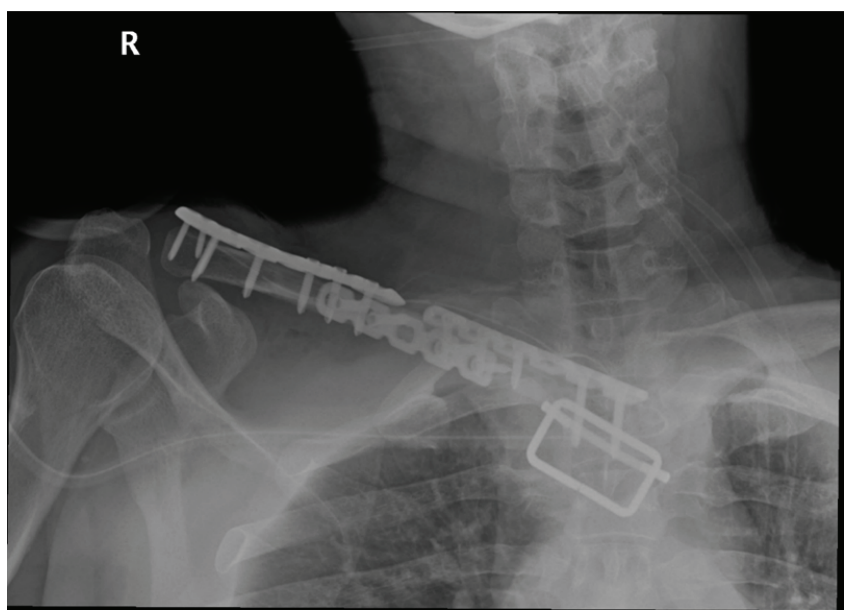

Fig. 4. After wide resection and allograft reconstruction, three plates and screws were fixed.

the hospital after experiencing discomfort at the operation site. The medial plate was fractured, but no further procedures were performed (Fig. 5). The range of motion after 28 months was active forward flexion $160^{\circ}$, abduction $130^{\circ}$, and external rotation 


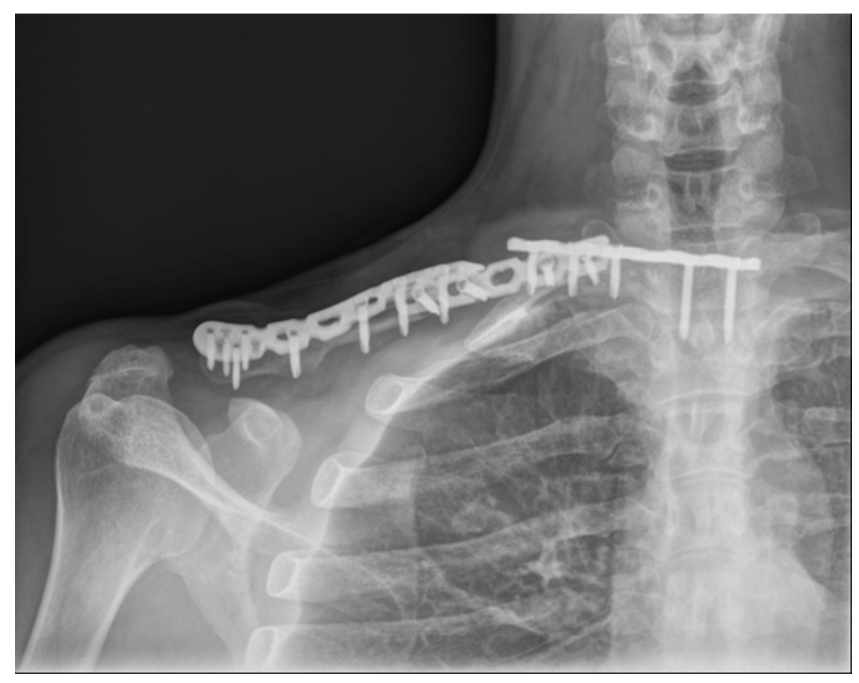

Fig. 5. X-ray taken at 23 months after surgery, showing that the fractured area at 11 months after surgery was well fused. An angular deformity and sternal screw loosening with medial plate pull-out were observed. Since there were no indications of additional pain or discomfort, further observation was performed.

at the side $80^{\circ}$. This was meaningful in that it indicated successful recovery of radiographic and functional outcomes after wide excision with allograft reconstruction for large parosteal osteoma of the clavicle.

\section{DISCUSSION}

Primary clavicle tumor and tumorous lesions are rare, and the majority of studies were case reports [2]. Since osteomas are benign lesions, it is important to differentiate them from malignant lesions like parosteal osteosarcoma or parosteal chondrosarcoma [3]. In our case, bone scan revealed an active bone lesion, and the clinical diagnosis of the radiology department was parosteal osteosarcoma due to suspicious involvement of the subscapularis enhancing soft tissue portion and deviating subclavian vein, making it difficult to determine treatment. For evaluation of soft tissue mass or bone tumor, biopsies with various techniques are helpful and important deciding factors. Choosing the appropriate biopsy is important for accurate diagnosis of tumors [4-6]. In our case, we questioned the accuracy of the biopsy since negative predictive values has been shown in the literature. The medial clavicular mass was diagnosed initially as an aneurysmal bone cyst, but due to its rapidly growing nature after diagnosis, open biopsy was performed, revealing a high-grade osteosarcoma [5]. In our case, the patient requested lesion removal regardless of pathology. Careful discussion was performed to determine whether or not a preoperative biopsy would be valuable. It was decided that wide excision be performed with negative margins without preoperative biopsy. In addition, the possibility of tumor seeding or contamination on the biopsy tract could not be ignored. Pressure on the surrounding tissues was an important factor in determining the type of surgery. After wide excision, a mass with a size of $4.9 \times 3.5 \times 3.5 \mathrm{~cm}$ with negative resection margins was obtained. Nora's lesion known as bizarre parosteal osteochondromatous proliferation was observed and was supportive of parosteal osteoma, but a clinicopathologic correlation was recommended. The final result was a parosteal osteoma and not a parosteal osteosarcoma as was suspected in preoperative MRI. In summary, the importance of biopsy in diagnosis of a tumor is well known. However, biopsy has the possibility of negative predictive values. Especially for the patient in our case, hoping for operative resection due to the large mass effect, the risk-benefit of biopsy must be considered.

Claviculectomy for clavicular tumor can be inferior functionally due to many issues such as loss of the role as a supporter, muscle weakness, cosmetic problems, and restriction of joint motion as well as loss of the protector effect for important vessels and nerves located in the back of the clavicle [7]. Performing reconstruction using allograft after resecting the clavicle can lead to many complications from the bone graft material. There is a way to re-insert the clavicle after radiation treatment to perform reconstruction [8]. In our case, satisfactory recovery of the radiographic and functional outcomes was obtained after wide excision with allograft reconstruction for large parosteal osteoma of the clavicle.

\section{ORCID}

Donghyup Shin

https://orcid.org/0000-0003-2282-0934

Wonseok Kim

https://orcid.org/0000-0002-4639-7088

Jungho Park

https://orcid.org/0000-0002-0641-8307

\section{REFERENCES}

1. Ren K, Wu S, Shi X, Zhao J, Liu X. Primary clavicle tumors and tumorous lesions: a review of 206 cases in East Asia. Arch Orthop Trauma Surg 2012;132:883-9.

2. Inokuchi T, Hitora T, Yamagami Y, Nishimura H, Yamamoto T. Parosteal osteoma of the clavicle. Case Rep Orthop 2014;2014: 824959.

3. Prabowo Y, Kamal AF, Kodrat E, Prasetyo M, Maruanaya S, Efar TS. Parosteal osteosarcoma: a benign-looking tumour, amenable to a variety of surgical reconstruction. Int J Surg Oncol 2020;2020:4807612.

4. Layfield LJ, Schmidt RL, Sangle N, Crim JR. Diagnostic accura- 
cy and clinical utility of biopsy in musculoskeletal lesions: a comparison of fine-needle aspiration, core, and open biopsy techniques. Diagn Cytopathol 2014;42:476-86.

5. Cundy WJ, Carter C, Dhatrak D, Clayer M. Primary osteosarcoma of the clavicle and the perils of bone biopsy. BMJ Case Rep 2015;2015:bcr2014208859.

6. Turkoz KH, Erol B, Seven IE. Tumor cell seeding in the biopsy tract and its clinical significance in osteosarcomas. J Surg Oncol 2018;118:1335-40.

7. Chen Y, Yu X, Huang W, Wang B. Is clavicular reconstruction imperative for total and subtotal claviculectomy? A systematic review. J Shoulder Elbow Surg 2018;27:e141-8.

8. Grimer RJ, Crockett SC. Extracorporeally irradiated clavicle as an autograft in tumour surgery. J Surg Case Rep 2015;2015:rju151. 